\title{
Vorwort zur zehnten Auflage
}

Die 10. Auflage wurde im Hinblick auf das MATLAB Release 2020a vollständig überarbeitet. Schwerpunkte dabei waren zum einen neue Funktionen zur Verarbeitung von Strings sowie zum Import und Export von Tabellen und Textdateien. Zum anderen wurden einige interaktive Werkzeuge deutlich überarbeitet, wie z.B. der Profiler und das Basic Fitting Tool. In der Signal Processing Toolbox wurde zudem der Signal Analyzer neu eingeführt. Das Ergebnis ist ein kompaktes Lehrbuch für den Einsteiger und gleichzeitig ein übersichtliches Nachschlagewerk für den fortgeschrittenen MATLAB-Anwender.

Neben den Beispielen, Übungsaufgaben und Lösungen steht auf der Internetseite www.matlabbuch.de auch wieder eine Bibliothek der Autoren mit nützlichen Extras für MATLAB und Simulink zur Verfügung. Diese Informationen finden sich auch auf der Homepage des De Gruyter Verlags www.degruyter.com.

Danken möchten wir zuallererst Herrn Professor i. R. Dierk Schröder für seine umfassende Unterstützung bei der Erstellung dieses Buches. Ausgehend von seiner Idee und seinem unermüdlichen Engagement, eine längst notwendige Vorlesung zum Softwarepaket Matlab und Simulink für Studenten der Fachrichtungen Energietechnik, Automatisierungstechnik und Mechatronik ins Leben zu rufen, konnten wir in sehr freier und kollegialer Arbeitsweise ein Skriptum zu dieser Vorlesung erstellen.

Seit dem ruhestandsbedingten Wechsel kann dieses Engagement unter Leitung von Herrn Professor Ralph Kennel, Ordinarius des Lehrstuhls für Elektrische Antriebssysteme und Leistungselektronik der Technischen Universität München, in vollem Umfang fortgesetzt werden. Für seine immer wohlwollende Unterstützung sowie für die vertrauensvolle und angenehme Zusammenarbeit danken wir ihm daher sehr herzlich.

Die äußerst positive Resonanz von Studenten unterschiedlichster Fachrichtungen sowie zahlreiche Anfragen aus Forschung und Industrie ermutigten uns, das ursprüngliche Skriptum einem größeren Leserkreis zu erschließen und als Buch zu veröffentlichen. Aufgrund der regen Nachfrage erscheint dieses Buch nun bereits in seiner 10. Auflage. Nicht zuletzt danken wir daher unseren zahlreichen Lesern, allen Dozenten, Studenten und Kollegen, die uns dabei mit ihren Anregungen und ihrer stets wohlwollenden Kritik unterstützten und noch unterstützen werden.

Für Verbesserungvorschläge und Hinweise auf noch vorhandene Fehler sind wir jederzeit dankbar und werden sie auf der Internetseite www.matlabbuch.de neben weiteren aktuellen Informationen rund um MATLAB veröffentlichen.

Dem De Gruyter Verlag danken wir für die Bereitschaft, dieses Buch zu verlegen. Besonderer Dank gilt hierbei Frau Skambraks für ihre hilfreiche Unterstützung und für die Übernahme des Lektorats.

München

Anne Angermann

Michael Beuschel

Martin Rau

Ulrich Wohlfarth 